\title{
ON THE MODELLING OF TWO-PHASE FLOW IN HORIZONTAL LEGS OF A PWR
}

\author{
D. BESTION* and G. SERRE \\ CEA, Grenoble, DEN-DM2S-STMF \\ 17 Rue des Martyrs, 38054, FRANCE \\ "Corresponding author. E-mail : dominique.bestion@cea.fr
}

Received November 20, 2012

This paper aims at presenting the state of the art, the recent progress, and the perspective for the future, in the modelling of two-phase flow in the horizontal legs of a PWR. All phenomena relevant for safety analysis are listed first. The selection of the modelling approach for system codes is then discussed, including the number of fluids or fields, the space and time resolution, and the use of flow regime maps. The classical two-fluid six-equation one-pressure model as it is implemented in the CATHARE code is then presented and its properties are described. It is shown that the axial effects of gravity forces may be correctly taken into account even in the case of change of the cross section area or of the pipe orientation. It is also shown that it can predict both fluvial and torrential flow with a possible hydraulic jump. Since phase stratification plays a dominant role, the Kelvin-Helmholtz instability and the stability of bubbly flow regime are discussed. A transition criterion based on a stability analysis of shallow water waves may be used to predict the Kelvin-Helmholtz instability. Recent experimental data obtained in the METERO test facility are analysed to model the transition from a bubbly to stratified flow regime. Finally, perspectives for further improvement of the modelling are drawn including dynamic modelling of turbulence and interfacial area and multi-field models.

KEYWORDS : Two-phase Flow Modelling, Stratification Criterion, System Code

\section{INTRODUCTION}

Hot Legs (HL), Cold Legs (CL), and Intermediate Legs (IL) of Pressurized Water Reactors (PWR) play an important role in accident sequences, particularly when twophase steam water flow occurs in the loops (see Figure 1 ). When the primary mass inventory is degraded in case of a Loss of Coolant Accident (LOCA) the phase coupling or decoupling controls the amount of water which is kept out of the Pressure Vessel and influences the occurrence of a Core uncovery with fuel clad temperature excursion. The formation of a liquid slug in IL after the end of natural circulation and the loop seal clearing may also induce a core uncovery. Many auxiliary circuits are connected to HL and CL such as safety injections, Residual Heat Removal circuit, and the pressurizer surge line. All these connections may also be considered as potential breaks when analysing the safety of the reactor.

The knowledge of the flow regime in horizontal pipes may play an important role in PWR accident scenarios. For example, during many LOCA transients, the flow regime in Hot Legs or Cold Legs influences the amount of liquid and steam lost at a break or entrained in the pressurizer surge line in case of pressure relief valve opening. Both the primary mass inventory and the primary pressure are very sensitive to the steam quality of the break or valve flow. As an example, the quality of the flow rate at a break or steam relief valve in the pressurizer will strongly depend

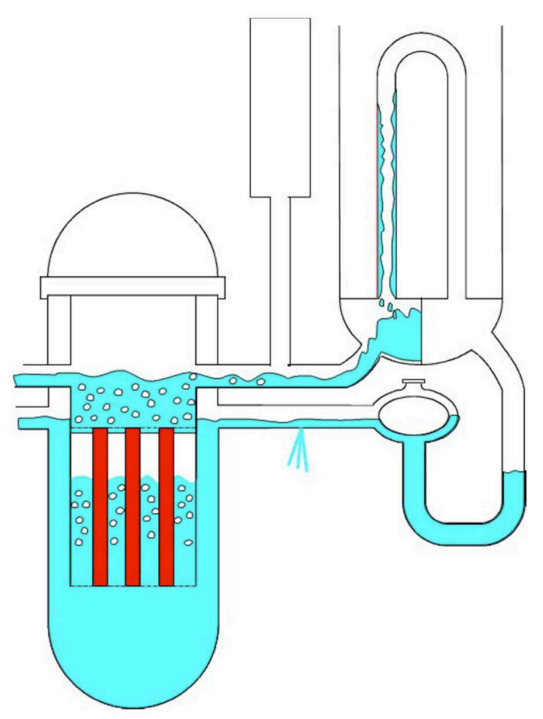

Fig. 1. Examples of Flow Situations Encountered in Hot Legs and Cold Legs in Accidental Transients of a PWR 


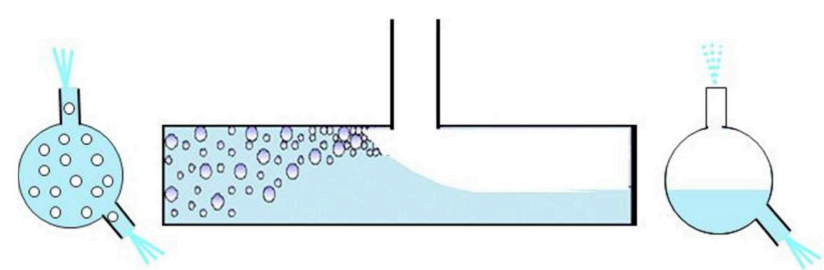

Fig. 2. Two Different Flow Regimes at the Pressurizer T Junction or at Two Different Break Orientations

on the flow regime at the $\mathrm{T}$ junction between $\mathrm{HL}$ and surge line as illustrated on Figure 2.

This paper aims at presenting the state of the art, the recent progress, and the perspective for the future, in the modelling of two-phase flow in horizontal legs of a PWR. All phenomena relevant for safety analysis are listed first. The selection of the modelling approach for system codes is then discussed, including the number of fluids or fields, the space and time resolution, and the use of flow regime maps. The classical two-fluid six-equation one-pressure model is then presented and its properties are described. This paper mainly refers to the CATHARE system code $[1,2,3]$ developed jointly by EDF, CEA, AREVA and IRSN, and some comparisons with other system codes [4, 5] are also made. Since phase stratification plays a dominant role, the Kelvin-Helmholtz instability and the stability of bubbly flow regime are discussed. A transition criterion based on a stability analysis of shallow water waves may be used to predict the Kelvin-Helmholtz insta-bility. Recent experimental data obtained in the METERO test facility are analysed to model the transition from a bubbly to stratified flow regime. Finally, perspectives for further improvement of the modelling are drawn including dynamic modelling of turbulence and interfacial area, and multi-field models.

\section{PHENOMENA OF INTEREST IN HOT LEG AND COLD LEG OF A PWR}

The main phenomenon in hot and cold legs is phase stratification, which dramatically decreases the mechanical coupling between phases. The interfacial friction in stratified flow is several orders of magnitude lower than in dispersed bubbly flow. Therefore, the entrainment of liquid water in the loop by steam coming from the core is minimized in stratified flow. This is a favourable feature since it keeps more water in the core without affecting the heat release to the Steam Generators (SG) which may continue even after the stop of two-phase natural circulation, by the reflux condensation mode. In the stratified flow regime in a horizontal pipe, there may be a non-horizontal free surface due to interfacial friction and/or due to some interfacial waves propagating at the free surface. In many Small Break Loss of Coolant accidents (SBLOCA) there is a liquid slug formed in the IL after the end of the two-phase natural circulation. This liquid slug is then removed by the socalled "loop seal clearing" process and some quantity of water is kept at the bottom of the IL, which depends on the interfacial friction at the free surface and on the predicted free surface slope which determines the minimum cross section for steam flow and then the maximum steam velocity. The slope of the free surface is the result of the interfacial friction and the slope influences the interfacial friction and the possible entrainment of water by determining the maximum steam velocity.

In the two-phase natural circulation mode, the flow at the entrance of the HL may be bubbly or stratified-bubbly -with a pure vapour flow at the top and a bubbly flow below the free surface- when the swell level in upper plenum decreases to the elevation of the HL. In most cases velocities are low and the flow regime changes progressively to pure stratified flow without any more bubbles.

In the case of stratified flow, waves are created at the free surface, which increase the surface roughness and the interfacial friction. Both fluvial and torrential flows may occur and a hydraulic jump may be created when there is a transition from torrential to fluvial regime.

In the case of reflux condenser mode, Counter Current flow limitation (CCFL) may occur in the Hot Leg in the zone of the bend and the SG entrance nozzle. Large amplitude waves reflecting on the bend were observed in some conditions in the MHYRESA tests [6]. CCFL may result from liquid entrainment at the crest of large amplitude waves.

The MHYRESA Hot-Leg entrainment tests [7, 8, 9] have also shown that for a given liquid level in the upper plenum, a hydraulic jump could be created and moved downstream when the gas flowrate is increased.

During the Reflooding of a Large Break LOCA (LBLOCA) a mist flow enters the HL and some deposition of droplets occurs. Liquid films are created along HL walls and a continuous liquid field is collected at the bottom of the pipe. Due to the large vapour velocity, droplets may be also entrained from the crest of waves. The mist flow which enters the SG tubes is the result of this deposition and entrainment of droplets occurring first in the upper plenum and in the HL. Prediction of the resulting droplet flow rate entering $\mathrm{SG}$ tubes is of prime importance for modelling the so-called "steam-bindind" effect [10]. The vaporization of droplets in the hot SG tubes creates additional pressure losses in the loops which decreases the flooding rate in the core.

In postulated accident sequences where pumps are supposed to operate in two-phase flow conditions, the phases may be well mixed at the outlet of the pump and may tend to stratification when flowing to the pressure vessel.

Postulated small and medium size breaks in HL, IL and CL may be oriented at the top, on the side or at the bottom of the pipe (see Figures 2 ). The quality and mass flowrate at the break depends a lot on the position of the break and on the flow regime. In a stratified flow there may be a very large difference between a bottom break, which 
entrains a large flowrate but a low quality, and a top break, which entrains a high steam flowrate and a low mass flowrate. Losing a high mass flowrate may induce a core uncovery with fuel clad temperature excursion. Having a high steam quality at the break induces a slower loss of primary mass and decreases primary pressure [11] so that actuation of safety injection will occur earlier. Therefore, core uncovery is less likely to occur and will be shorter than in the bottom break case. This shows the importance of predicting the flow regime and more generally the vertical repartition of steam and water in horizontal pipes.

Postulated loss of Residual Heat Removal (RHR) accidental transients at very low pressure (close to atmospheric pressure) may create high velocity steam flow in the HL. Such situations may occur during mid-loop operation with vent valves possibly open or even with an open manhole in the pressurizer or the SG header. The loss of mass inventory is controlled by water entrainment either in the pressurizer or to the SG, which depends a lot on the flow regime in the HL. Transitions from stratified to slug flow by KelvinHelmholtz instability or to stratified-mist flow by droplet entrainment at wave crests may occur and influence the water entrainment out of the circuit.

Safety injection in CL, or in both CL and HL, induce Direct Contact Condensation since subcooled water is injected into a saturated steam or steam-water mixture. Analysis of safety injection COSI experiments (see Janicot \& Bestion [12]) has shown that a stratified flow is created in most cases. However, the high turbulence created by SI jets induces an efficient mixing which enhances the local condensation. In the case of a very high condensation rate (when the SI flowrate is very high) in a CL there may be a condensation induced Kelvin-Helmholtz instability with a liquid slug which may go back to the pump at high velocity. UPTF refill tests have also shown that condensation may be sensitive to the presence of small amounts of degassed nitrogen at the accumulator injection, which limits the condensation and prevents large instabilities.

In summary the main phenomena that must be predicted with sufficient accuracy in horizontal legs are:

- Transition from bubbly to stratified flow

- Transition from stratified flow to slug flow by KelvinHelmholtz instability

- Transition from stratified flow to stratified-mist flow by droplet entrainment

- Transition from mist flow to stratified-mist flow by droplet deposition

- Presence of free surface waves which increase the interfacial friction

- Occurrence of fluvial and torrential flow regimes with a possible hydraulic jump

- Effects of the cross section area changes and of orientation changes (in the bends of HL and IL) on the free surface position

- All phenomena which affect a possible free surface inclination, such as interfacial friction or wave propagation

- Remaining water mass in IL after a loop seal clearing

- Interfacial heat and mass transfers associated with DCC at safety injections, which depend a lot on turbulence created by ECCS jets

Other phenomena exist in horizontal legs which are less sensitive in accidental transients. Wall friction in large diameter pipes is rather small compared to friction in other components such as the core and SGs so they do not influence the loop flowrate a lot. Therefore the modelling of wall friction does not require a high accuracy. In bubbly or slug flows in horizontal legs there is practically no slip between gas and liquid due to the absence of buoyancy forces, so the transition from bubbly to slug flow and the interfacial friction in these flow regimes do not require very accurate modelling.

\section{SELECTING A MODELLING APPROACH}

\subsection{1-D Modelling Versus 3D Modelling}

All two-phase flows are 3D flows since all flow parameters (velocities, phase volume fractions, pressure, and temperatures) depend on the three space directions but 1D modelling is possible when there is a privileged direction of velocity as it is in pipe flow. This is the reason why all system thermalhydraulic codes (see $[1,2,3,4,5]$ ) model two-phase flow in pipes with a 1D approach. Multi-dimensional models are only required when a $3 \mathrm{D}$ velocity field is encountered, like the downcomer refill in a LBLOCA or the transverse mixing phenomena encountered in a reactor core when there is a transverse power profile.

The phenomena of interest in the horizontal legs of a PWR listed above clearly show the interest of predicting not only the average void fraction in a cross section but also the vertical repartition of phases which tends to be non-uniform due to transverse gravity force. This vertical repartition may strongly influence the break flow and consequently the total primary mass inventory and the primary pressure evolution, but also the mass repartition in the circuit and consequently the severity of a possible core uncovery. Therefore two approaches are possible:

1. Simulating two-phase flow in HL, CL and IL with 3D CFD which in principle predicts 3D fields of all flow parameters

2. Using 1D modelling with sufficient characterization of the various flow regimes so that an approximate vertical phase repartition may be predicted

3D CFD was used by Murase et al. [13] to investigate CCFL in HL. The objective was to validate the two-phase CFD tool on existing data at reduced scale and in UPTF scale 1 data, and then to use the same simulation tool to extrapolate to other pressure conditions and other reactor designs. However, in the end, a CCFL correlation is derived from CFD simulation which will be used in system codes 
for simulating accidental transients. Therefore CFD is used in a multi-scale analysis methodology.

Such multi-scale analysis is now possible for some applications as shown by Bestion [14]. However, the twophase CFD is not yet very mature [15]; it cannot be applied to all flow regimes $[16,17]$, and it requires a very high CPU cost. The classical TH system codes will remain the only way to simulate many reactor transients for a long time. Therefore it is still relevant to try to improve the capabilities of 1-dimensional modelling of horizontal two-phase flow.

\subsection{Space and Time Resolution of the Modelling}

The 1-D two-fluid models used in most system codes are the result of space averaging (see Vernier \& Delhaye, [18]) or composite space and time averaging (Delhaye et al. [19]).

Using space averaging over a cross section of the duct or over a slice of the duct is necessary to reduce the problem to a 1-D formulation. Using time averaging (Ishii, [20]) over a time interval [T] filters turbulent fluctuations and flow fluctuation due to phase intermittency. This is in principle only possible when the largest fluctuations have a time scale small enough compared to the overall flow evolution. However in all situations encountered in accidental transients one cannot find a time scale which can clearly separate fluctuations from mean flow evolutions. This is one of the main weaknesses of current 1-D models of system codes, which cannot specify the time scale of filtered processes. This time scale does not affect the formulation of the left hand side terms of the balance equations (differential terms for time variations and advection terms) but it may affect the formulation of source terms for wall and interfacial transfers. The weakness is particularly important when dealing with stratified flow and transition to slug flow by Kelvin-Helmholtz instability.

The most favourable case is a quasi-steady stratified flow. In such a case the time scale for mean flow variations can be much larger than all turbulent fluctuations and void fraction fluctuations due to the presence of waves. In such a case, waves are not predicted but their presence should be taken into account in the formulation of interfacial transfers. This is a very pleasant feature for system codes since large time steps are possible and relatively long meshes are also possible - both features allow low CPU cost- without affecting the quality of predictions too much. The prediction of a possible hydraulic jump is still possible since it will be steady or quasi-steady.

Now, if the gas flowrate is increased up to a value which makes some wavelength unstable there should be a liquid bridging and a transition to the slug flow. Such transition is necessarily a transient process with small time scales. This transient process cannot be predicted by a time averaged equation unless the time interval $[\mathrm{T}]$ is very small. I Tiselj $[21,22]$ has shown that for predicting the Condensation Induced Water-Hammer (CIWH) with the two-fluid model, classical flow regime maps cannot be used as they are developed for established and steady-state flow regimes, and existing slug flow correlations are not directly applicable as they give time-averaged heat, mass and momentum transfers instead of the instantaneous local values needed for the description of the transient process. A single slug was explicitly followed in the CIWH simulation with a 1D two-fluid model and it required an explicit modelling of slug formation and slug propagation.

One can conclude that the two-fluid approach has the capability to predict both quasi-steady horizontal flows and rapid transients, but current system codes only have closure laws for the former case. Such a limitation may not be a problem if it is clearly identified but the limit between transients which can be predicted and those which cannot is not fully clear.

\subsection{The Two-fluid Model or the Multi-field}

The two-fluid model is used in current system codes and has proved its capability to predict phase coupling or decoupling depending on the flow regime. As will be shown later the transitions from bubbly to stratified and from stratified to slug by Kelvin-Helmholtz instability can also be modelled. The characteristics of fluvial and torrential flows with possible hydraulic jumps can also be described.

The multi-field models have better capabilities only for complex flow regimes:

- Stratified-mist flow will be much better modelled by a model with two liquid fields (droplets + continuous liquid) since the velocities of the two liquid fields are treated separately

- Stratified-bubbly flow will be much better modelled by a model with two gas fields (bubbles + continuous gas) since the velocities of the two gas fields are treated separately

\subsection{Turbulence and Interfacial Area Modelling}

All interfacial transfers depend on the interfacial area, and turbulence has strong interactions with the presence of interfaces. Turbulence may break bubbles or drops and may also favor coalescence. Friction along interfaces and in the wake of bubbles and drops creates additional turbulence. In current system codes only algebraic models of turbulence and interfacial area are used. It means that no dynamic evolution of interfacial areas and turbulence with associated relaxation times may be described. Some local equilibrium must be assumed, such as production equal dissipation of turbulence or coalescence equal break up, to obtain algebraic models. The first attempts to develop coupled turbulence and interfacial area dynamic modeling were restricted to bubbly-slug flows in vertical pipes.

It is clear that the algebraic models have limitations and that dynamic modeling with transport equations may better describe some non-established flow conditions. This may be particularly useful when a bubbly flow enters a horizontal pipe (IC) and a progressive transition to stratified flow occurs which depends on the evolution of bubble 
coalescence and of the turbulence intensity which is not yet well established. One must keep in mind that fully established flows in pipes only exist after at least 50 diameters distance from the entrance. Horizontal legs in PWRs never exceed 10 to 20 diameter lengths which means that all flows in HL, IL and CL are non-established flows and a dynamic modeling of interfacial area and turbulence may be a way to improve the accuracy of predictions.

\subsection{The use of Flow Regime Maps}

System codes often use Flow regime maps to decide which flow regime is present in order to apply the adequate interfacial transfer models. Many academic studies were devoted to horizontal flow regimes [23-28] in air-water conditions. Most of these investigations tried to use well established flow regimes in rather long pipes. One should then more properly speak of "Established Flow regime maps", since a stratified flow may need a very long pipe to be fully established. If gas and liquid enter a pipe with some imposed velocities, the equilibrium slip is reached when there is equilibrium between wall friction forces and interfacial friction (see the equations in section 4). Since the interfacial friction is low in stratified flow, acceleration or deceleration of phases will be a slow process before reaching the equilibrium value of the level $\mathrm{H}$ and of the slip ratio. It was shown by Barnea et al $[27,28]$ that the stratified domain depends a lot on a small inclination angle of the pipe with a horizontal axis. It was also observed in MHYRESA tests [6-9] that downstream boundary conditions may play an important role on stratified flow, creating either fluvial or torrential flow. In the case of a fluvial flow regime, the downstream water level is imposed and there is no possible established flow for all entrance water levels, which are different from the outlet level. Moreover, in the case of fluvial downstream boundary conditions and torrential inlet conditions, a hydraulic jump is created in the pipe and the flow may be steady but non-established. Hot legs and the bottom of intermediate legs have upward bends at the downstream end and flow is likely to either be fluvial or torrential then fluvial. In cold legs, when the water level in the pressure vessel is low, a stratified flow in the cold leg is likely to be torrential or just critical.

When the limit of stratified flow is plotted in a Jl versus JV plane (see Bestion \& Micaelli, [29]), it is often the result of a stratification criterion which gives a relation between $\mathrm{Jl}, \mathrm{Jv}$ and the void fraction $\alpha$, combined with a force balance relation which gives a second relation between $\mathrm{Jl}, \mathrm{JV}$ and $\alpha$. The force balance is the result of the assumption that the flow is established. Therefore the domain of stratification in the plane $\mathrm{Jl}$ versus $\mathrm{Jv}$ represents only the domain of "Established Stratified Flow", which should not be compared to the stratified flow domain in non-established flow as often encountered in the horizontal legs of PWRs. However, the transition criterion itself may remain valid in non-established flow.

\section{DERIVATION OF TWO-FLUID EQUATIONS}

Having in mind that the main difficulty in horizontal legs is to correctly predict the stratification occurrence and the characteristics of stratified flow, attention was paid in the CATHARE code development to derive the best possible system of equations.

The CATHARE code two-fluid 6-equation model able to describe stratified flows was derived by de Crecy [30] who also analyzed its properties. Assuming:

- A hydrostatic transverse pressure gradient

- Vapor and liquid densities uniform over the cross section

- Continuity of pressure across the interface (no surface tension effect and no pressure jump induced by mass transfer)

Area averaging of local instantaneous equations multiplied by the phase indicator function for liquid and /or vapor leads to the following momentum phase $\mathrm{k}$ equation projected on the curvilinear abscissa $z$ of the pipe:

$$
\begin{aligned}
& \frac{\partial}{\partial t} \mathrm{~A} \alpha_{k} \rho_{k} V_{k}+ \frac{\partial}{\partial z} \mathrm{~A} \alpha_{k} \rho_{k} V_{k}^{2}+A \alpha_{k} \frac{\partial P}{\partial z} \\
&=\mathrm{A} \alpha_{k} \rho_{k} g_{z}+A \varepsilon_{k} \Gamma W_{i}-\varepsilon_{k} S_{i} \tau_{i}-S_{k} \tau_{w k} \\
& \quad-A p_{i} \frac{\partial \alpha_{k}}{\partial z}-A \varepsilon_{k} G_{z}-D_{k}-\alpha_{k} \Delta_{k} \frac{\partial A}{\partial z}
\end{aligned}
$$

with:

A : cross section area

$\mathrm{P}:$ Average pressure in the cross section

$$
P=\frac{1}{A} \sum_{k} A_{k} P_{k} d A_{k}
$$

$\mathrm{g}_{\mathrm{z}}$ : axial component of the gravity acceleration vector (along curvilinear abscissa $\mathrm{z}$ )

$$
g_{z}=-g \sin \theta
$$

$\epsilon_{v}=1$

$\epsilon=-1$

$\Delta_{k}$ pressure difference between the average pressure $P_{k}$ in phase $\mathrm{k}$ and pressure at the interface $\mathrm{Pi}$

$$
\begin{gathered}
\Delta_{k}=-\rho_{k} g \sin \theta\left(X_{k}-X_{i}\right) \\
p_{i}=\alpha \Delta_{l}-(1-\alpha) \Delta_{v}+\alpha(1-\alpha) \frac{\partial\left(\Delta_{v}-\Delta_{l}\right)}{\partial \alpha} \\
G_{z}=\alpha(1-\alpha) \frac{\partial\left(\Delta_{v}-\Delta_{l}\right)}{\partial z} \\
D_{k}=\int_{S_{k}} \overrightarrow{n_{z}} \cdot \overrightarrow{n_{k}}\left[P_{k}(x, y, z)-P_{i}\right] \frac{d s}{\overrightarrow{n_{k}} \cdot \overrightarrow{n_{k s}}}
\end{gathered}
$$

For each phase $\mathrm{k}, \mathrm{D}_{\mathrm{k}}$ takes into account the pressure difference due to gravity between the interface and the wall perimeter in contact with phase $\mathrm{k}$. 
$D_{k}$ and $\Delta_{k}$ and terms depend on the pipe shape. In the particular case of a square duct, the momentum equation is written:

$$
\begin{aligned}
& \frac{\partial}{\partial t} \mathrm{~A} \alpha_{k} \rho_{k} V_{k}+\frac{\partial}{\partial z} \mathrm{~A} \alpha_{k} \rho_{k} V_{k}^{2}+A \alpha_{k} \frac{\partial P}{\partial z}=\mathrm{A} \alpha_{k} \rho_{k} g_{z}+A \varepsilon_{k} \Gamma W_{i}-\varepsilon_{k} S_{i} \tau_{i} \\
&-S_{k} \tau_{w k}-A p_{i} \frac{\partial \alpha_{k}}{\partial z}+\frac{1-\alpha_{k}}{4} p_{i} \frac{\partial A}{\partial z} \\
&+A^{3 / 2} \varepsilon_{k} \alpha(1-\alpha) \frac{\rho_{m}}{2} \frac{\partial g \cos \theta}{\partial z}
\end{aligned}
$$

with:

$$
\begin{gathered}
p_{i}=\alpha(1-\alpha)\left(\rho_{L}-\rho_{V}\right) g \cos \theta A^{1 / 2} \\
\rho_{m}=\alpha \rho_{V}+(1-\alpha) \rho_{L}
\end{gathered}
$$

The expression for $p_{i}$ in a circular pipe should include a shape function of void fraction which is often forgotten and replaced with 1 .

In the CATHARE code 1-D module, $p_{i}$ is written:

$$
p_{i}=\alpha(1-\alpha)\left(\rho_{L}-\rho_{V}\right) g \cos \theta D
$$

The three last terms of the momentum equation are specific to stratified flows and represent axial effects of gravity on axial momentum in the case of axial variation of a pipe section, of the water level, and of pipe inclination.

The first of these three terms controls the characteristic velocity corresponding to void fraction waves which are shallow water waves at the free surface of the stratified flow.

The elimination of the pressure gradient from the two momentum equations provides a "cross momentum equation" which controls the difference between the phasic velocities.

$$
\begin{gathered}
\frac{\partial}{\partial t} \mathrm{~A}\left[\alpha_{v} \alpha_{l} \rho_{v} V_{v}-\alpha_{v} \alpha_{l} \rho_{l} V_{l}\right]+\frac{\partial}{\partial z} \mathrm{~A}\left[\alpha_{v} \alpha_{l} \rho_{v} V_{v}^{2}-\alpha_{v} \alpha_{l} \rho_{l} V_{l}^{2}\right] \\
=-\mathrm{A} \alpha(1-\alpha)\left(\rho_{l}-\rho_{l}\right) g_{z}+A \Gamma W_{i}-S_{i} \tau_{i}-\alpha_{l} S_{v} \tau_{w v} \\
+\alpha_{v} S_{l} \tau_{w l}-A p_{i} \frac{\partial \alpha_{k}}{\partial z}+\frac{1-2 \alpha}{4} p_{i} \frac{\partial A}{\partial z} \\
+A^{3 / 2} \alpha(1-\alpha) \frac{\rho_{m}}{2} \frac{\partial g \cos \theta}{\partial z}
\end{gathered}
$$

The properties of the momentum equations in stratified flow are illustrated in Figure 4 below where water is injected very slowly at the entrance of a pipe with area restriction
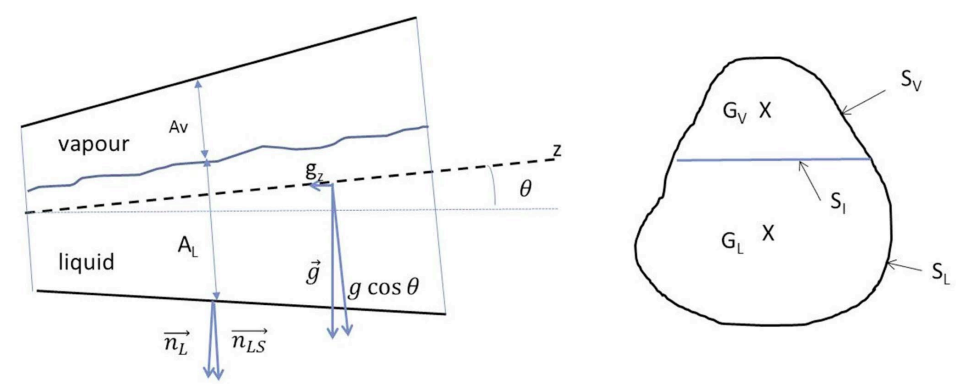

Fig. 3. Notations used in Momentum Equations
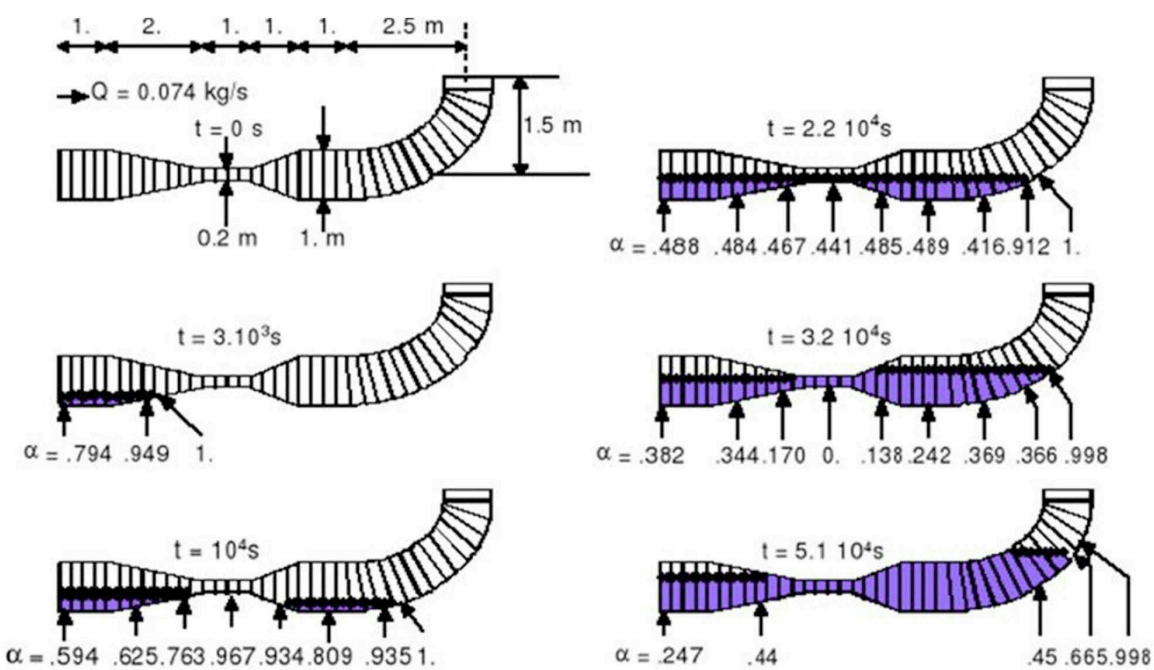

Fig. 4. Liquid Level at Rest in a Pipe with Varying Diameter and Inclination. 
and enlargement followed by a bend. Gas velocity is zero at the entrance and pressure is imposed at the outlet. It is shown that the water level plotted from the predicted void fraction respect a horizontal free surface even in varying diameter zones and in the bend. This is the result of the 3 last terms of the momentum equations.

The "cross-momentum equation" obtained by eliminating the pressure gradient between gas momentum and liquid momentum controls the slip ratio, whereas the total mixture momentum equation (addition of the gas and liquid momentum equation) controls relations between flowrate and axial pressure gradient.

In the case of horizontal steady established flow (d/dt $=0$ and zero axial gradient of velocities and void fraction) and for a constant cross section horizontal pipe without interfacial mass transfer, one can write a simple force balance representing the equilibrium:

$$
S_{i} \tau_{i} \cong \alpha S_{L} \tau_{w L}-(1-\alpha) S_{V} \tau_{w V}
$$

As explained above, this equilibrium is practically never reached in HL, IL and CL but it may be used to compare the code with "established flow regime maps".

In many stratified situations in HL, IL and CL, wall friction terms are negligible but there is a level slope such that the force balance may be described by the following approximation equation:

$$
S_{i} \tau_{i} \cong-A p_{i} \frac{\partial \alpha_{k}}{\partial z}
$$

In the case of a stationary steep level slope such as a hydraulic jump, the inertial forces may not be negligible and the situation may be described by the following approximated equation:

$$
\frac{\partial}{\partial z} \mathrm{~A} \alpha_{v} \alpha_{l}\left[\rho_{v} V_{v}^{2}-\rho_{l} V_{l}^{2}\right]+S_{i} \tau_{i} \cong-A p_{i} \frac{\partial \alpha_{k}}{\partial z}
$$

\subsection{Properties of the System of Equations}

As shown by de Crecy [30], the characteristic equation may be written from mass and momentum equations for gas and liquid.

$$
\lambda^{2}-2 \lambda \widetilde{\rho V}+\rho \widetilde{V}^{2}-p_{i}=0
$$

The hyperbolicity condition is:

with

$$
p_{i}>\rho \widetilde{V}^{2}-\frac{(\widetilde{\rho V})^{2}}{\rho_{m}}
$$

$$
\tilde{X}=\alpha X_{L}+(1-\alpha) X_{V}
$$

or:

$$
p_{i}>\frac{\alpha(1-\alpha) \rho_{v} \rho_{l}\left(V_{v}-V_{l}\right)^{2}}{\rho_{m}}
$$

The characteristic velocities are $\mathrm{W}+\mathrm{C}_{\alpha}$ and $\mathrm{W}-\mathrm{C}_{\alpha}$ with:

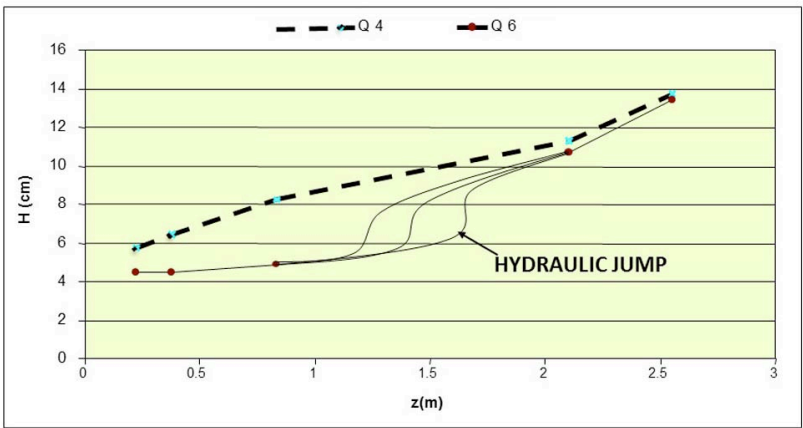

Fig. 5. Examples of Water Level Evolution in the MHYRESA Hot Leg

$$
\begin{aligned}
C_{\alpha}^{2} & =\frac{\rho_{m} p_{i}-\alpha(1-\alpha) \rho_{v} \rho_{l}\left(V_{v}-V_{l}\right)^{2}}{\rho_{m}^{2}} \\
W & =\frac{\alpha \rho_{l} \quad V_{l}+(1-\alpha) \rho_{v} \quad V_{v}}{\alpha \rho_{l}+(1-\alpha) \rho_{v}}
\end{aligned}
$$

The limit between fluvial (subcritical with respect to void waves) and torrential (supercritical with respect to void waves) occurs when $\mathrm{W}=\mathrm{C}_{\alpha}$, or when:

$$
p_{i}=\widetilde{\rho V^{2}}
$$

Then depending on the value of $p_{i}$, one may find non hyperbolic flow, torrential flow or fluvial flow as below:

$$
\begin{array}{rlrl}
\text { Non hyperbolic torrential fluvial } & \text { fla } \\
\rho \widetilde{V}^{2}-\frac{(\widetilde{\rho V})^{2}}{\rho_{m}} & \rho \widetilde{V}^{2} & p_{i}
\end{array}
$$

An example of two flows obtained in the MHYRESA test facility (representing a PWR hot leg) is shown in Figure 5. The water level $\mathrm{H}$ increases progressively in the case of the flowrate Q4, whereas it increases suddenly with the flowrate Q6. Condition Q4 is fluvial from entrance to exit whereas condition Q6 is torrential then fluvial after a hydraulic jump.

Momentum equations with the $p_{i} \partial \alpha / \partial z$ term presented above have the capability of the Saint-Venant equations to distinguish the two conditions and to predict a hydraulic jump.

\section{KELVIN-HELMHOLTZ ANALYSIS}

All models for the transition from stratified flow to slug flow or intermittent flow are based on an analysis of the Kelvin-Helmholtz instability in one or two dimensions. The CATHARE model assumes that a one-dimensional analysis is sufficient and that the model equations contain enough information to study these instabilities.

Two kinds of linear stability analysis have been investigated [29]: a high frequency linear stability and a marginal stability analysis. They consist in determining the conditions 


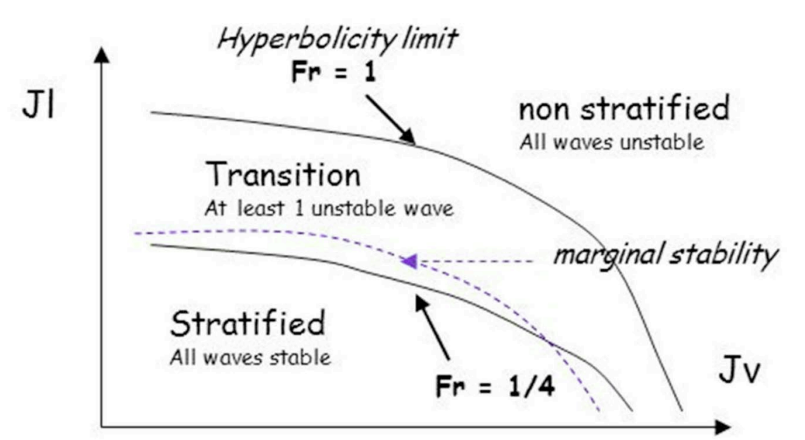

Fig. 6. Qualitative View of Stability Domains with Respect to Kelvin-Helmholtz

when a void fraction wave has a positive growth rate.

High frequency analysis considers only differential terms of the equation and neglects the viscous and algebraic source terms. It corresponds to the hyperbolicity limit of the system of equations. It can be demonstrated that when the hyperbolicity is not satisfied, high frequency waves are unstable and all wave lengths are unstable. The limit overestimates the stability domain.

It corresponds to a limiting Froude number defined as:

$$
F r=\frac{\rho_{v} \rho_{l}\left(V_{v}-V_{l}\right)^{2}}{g \cos \theta\left(\rho_{l}-\rho_{v}\right) \rho_{m} D}=1
$$

The marginal stability analysis takes into account all terms of the equation and determines the limit at which at least one wavelength becomes unstable. There is no explicit expression for this limit and it must be solved numerically [29].

The two limits are illustrated in the plane $\mathrm{Jv}$ versus $\mathrm{Jl}$ by adding the mechanical equilibrium equation as in Figure 6. It was observed that the limit value of 0.25 for the Froude number is close to other stratification criteria (Taitel and Dukler, [26]) and not far from the marginal stability limit.

Therefore it was considered that:

$$
\begin{array}{lll}
1<\mathrm{Fr} & \text { non stratified flow } & (\mathrm{R} 1=0) \\
0.25<\mathrm{Fr}<1 & \text { transition } & (0<\mathrm{R} 1<1) \\
\mathrm{Fr}<0.25 & \text { stable stratification } & (\mathrm{R} 1=1)
\end{array}
$$

A rate of stratification $\mathrm{R} 1$ in the range 0 to 1 may be defined based on the K-H instability.

However, this only considers the stability of a stratified flow; it is not sufficient to determine the flow regime. The stability of a bubbly flow regime must also be analysed.

\section{STABILITY OF BUBBLY FLOW REGIME}

\section{The main physical phenomena}

A horizontal bubbly flow can remain dispersed bubbly or change to another flow regime depending on the forces acting on the bubbles in the vertical direction:

- The buoyancy force may induce bubble rising and accumulation at the upper part of the pipe, where they can coalesce. If the coalescence dominates, the bubbles will merge leading to large gas pockets that eventually lead to stratified flow, slug flow or plug flow.

- Turbulence of the liquid tends to mix the bubbles and homogenizes the bubble concentration, preventing their accumulation at the upper part of the pipe and thus their coalescence. Bubbles are dispersed by being entrained by the liquid eddies due to the drag force and added mass force associated with the fluctuating velocity field.

- Average drag force in the mean flow slows the movement of the bubbles whatever the direction.

Therefore, the persistence of the dispersed flow will strongly depend on the liquid turbulence intensity since it can first prevent the bubbles rising, then prevent their coalescence and it may also break the largest ones. It also depends on the bubble size: small bubbles are more likely to be entrained by eddies since the drag coefficient is higher than for large bubbles. In addition, the buoyancy force is proportional the bubble size to the cube when drag and turbulent dispersion forces are proportional to the squared bubble size.

\section{Modelling the transition}

A first approach in the modelling of the transition from bubbly to stratified flow or slug flow consisted in comparing two velocity scales: $\mathrm{Vb}$ is the bubble rising velocity and $\mathrm{Vt}$ is the liquid turbulent velocity scale. One will consider that the flow will remain bubbly if the turbulent velocity is significantly larger than the rising velocity and transition to stratification may occur in the opposite situation. Therefore, three steps have to be realized in this approach:

1. Modelling the bubble rising velocity. It can be estimated by the terminal velocity for a single bubble.

2. Modelling the turbulent velocity. Several ways can be explored to estimate it:

- to find a relation between this velocity and the mean flow velocity or the wall friction velocity,

- to use an algebraic turbulence model,

- writing a transport equation for the turbulent kinetic energy and then to use the square root of this quantity as an estimation of the turbulent velocity.

3. Defining a threshold for the ratio of these velocities corresponding to the transition, using experimental data.

\section{Bubble rising velocity}

The terminal velocity of a bubble in an infinite medium at rest is obtained balancing drag (right hand side) and buoyancy (left hand side) forces:

$$
\frac{1}{6} \delta^{3} \pi g\left(\rho_{L}-\rho_{g}\right)=\frac{1}{8} \pi \delta^{2} C_{d} \rho_{L} V_{b}^{2}
$$

The velocity is then:

$$
V_{b}=\sqrt{\frac{4}{3} \delta \frac{g\left(\rho_{L}-\rho_{g}\right)}{C_{d} \rho_{L}}}
$$


$\delta$ and $C_{d}(\delta)$ have to be expressed to close the problem.

In the CATHARE 2 code, for simplicity's sake the distorted bubble drag coefficient has been chosen as a function of the Laplace scale $£$ :

$$
\begin{gathered}
C_{d}=\frac{2}{3} \frac{\delta}{£} \\
£=\sqrt{\frac{\sigma}{g\left(\rho_{L}-\rho_{g}\right)}}
\end{gathered}
$$

The main advantage of this choice is that the bubble diameter completely disappears in the bubble velocity expression:

$$
V_{b}=\sqrt{2 £ \frac{g\left(\rho_{L}-\rho_{g}\right)}{\rho_{L}}}
$$

Therefore, the rising velocity depends only on the thermodynamic properties of the fluid:

$$
V_{b}=\left(\frac{4 \sigma g\left(\rho_{L}-\rho_{g}\right)}{\rho_{L}^{2}}\right)^{1 / 4}
$$

To generalise the velocity to all types of bubbles, various correlations for the drag coefficient should be used. But the bubble diameter would become an unknown of the problem. This point is discussed later.

\section{Turbulent velocity}

In the CATHARE 2 code [1], no dynamic turbulence model is available and only an algebraic expression of the turbulent velocity scale is possible. Then it was considered that the turbulent liquid velocity is proportional to the wall friction velocity:

$$
V_{t} \approx V_{f}=\sqrt{\frac{\tau_{w L}}{\rho_{L}}}=\sqrt{\frac{C_{f}}{2}} V_{L}
$$

Using a constant friction coefficient corresponding to the lower limit in the CATHARE friction model:

$$
C_{f}=0.003
$$

One obtains:

$$
V_{t} \approx \sqrt{\frac{0.003}{2}} V_{L} \approx 0.039 V_{L}
$$

In CATHARE 3, an original algebraic turbulence model has recently been developed for single-phase flow (Chandesris et al [31]). It gives the spatially averaged value of the turbulent kinetic energy in established flows:

$$
k_{\infty}=c_{1 \infty} V_{L}^{2} C_{f}^{2 / 3}
$$

In pipe flows, the Blasius friction model $C_{f}=0,097 / \mathrm{Re}_{H}^{1 / 4}$ gives:

$$
k_{\infty}=c_{2 \infty} V_{L}^{2} \operatorname{Re}_{H}^{-1 / 6}
$$

With the constant: $c_{2 \infty}=0.0367$

Then, the turbulent velocity is estimated as:

$$
V_{t}=c_{3 \infty} V_{L} \operatorname{Re}_{H}^{-1 / 12}
$$

With the constant: $c_{3 \infty}=\sqrt{0.0367} \approx 0.19$

\section{Thresholds for the transitions}

The flow map criteria are written as a function of the ratio of the two velocities.

In the former $\mathrm{Vt}$ expression the ratio is:

$$
\frac{V_{t}}{V_{b}}=C \mid V_{L}\left(\frac{\sigma g \Delta \rho}{\rho_{L}^{2}}\right)^{-1 / 4}
$$

With $C=\sqrt{0.003 / 4}$

Finally, the constant $\mathrm{C}$ was omitted in the dimensionless velocity $\mathrm{V}^{*}$ for various reasons: both turbulent and bubble rising velocities are only approximations and, moreover, the thresholds for the transitions will in fact integrate this constant and the approximations:

$$
V^{*}=\mid V_{L}\left(\frac{\sigma g \Delta \rho}{\rho_{L}^{2}}\right)^{-1 / 4}
$$

With the new Vt expression, the ratio of the two velocities is slightly different from the previous one since it depends on the Reynolds number:

$$
V^{*}=\frac{V_{t}}{V_{b}}=c_{3 \infty} \operatorname{Re}_{H}^{-1 / 12}\left|V_{L}\right|\left(\frac{\sigma g \Delta \rho}{\rho_{L}^{2}}\right)^{-1 / 4}
$$

Since the models for both rising and turbulent velocities are supposed to give only the right order of magnitude, some data are requested to adjust the value of the velocity ratio at which transition will occur.

Two experimental data sources have been used to calibrate the transition criterion:

- Super Moby-Dick (SMD) is a high pressure steamwater loop. It was the first database used to set the thresholds (see Bestion [1]). Vertical profiles of steamwater mixture density, obtained by X-Ray tomography, were used to recognise the flow regimes.

- Recently the METERO experiment provided new data at atmospheric pressure in air-conditions. Thanks to a Plexiglas test section, the flow regimes were directly observed. Moreover, turbulent velocities were measured to validate the assumptions made on the turbulent velocity scale.

\section{Data from the Super Moby Dick test facility}

The first data were obtained in the Super Moby Dick test facility (Memponteil) [32] in the pressure range 20 to 100 bars with an $80 \mathrm{~mm}$ inner diameter horizontal test section (see Figure 7) with variable mass flow rate, quality and pressure. A 30-beam X-ray densitometer was used to obtain vertical profiles of the steam-water mixture density. Examples of typical profiles are given in Figure 8. Averaged void fractions as well as collapsed levels Hc were obtained by data processing. The parameter $\mathrm{C}_{\text {STRAT }}$ was defined to characterize a "rate of stratification" in the test section:

$$
C_{\text {STRAT }}=\frac{\rho^{-}-\rho^{+}}{\rho_{L}-\rho_{V}}
$$




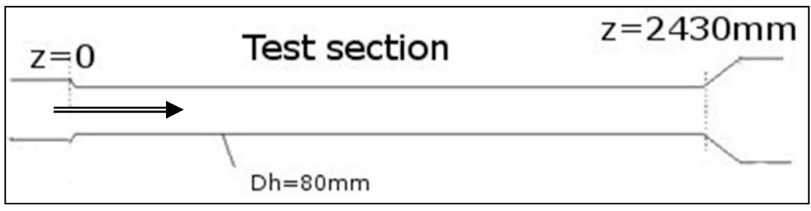

Fig. 7. Super Moby-Dick Test Section

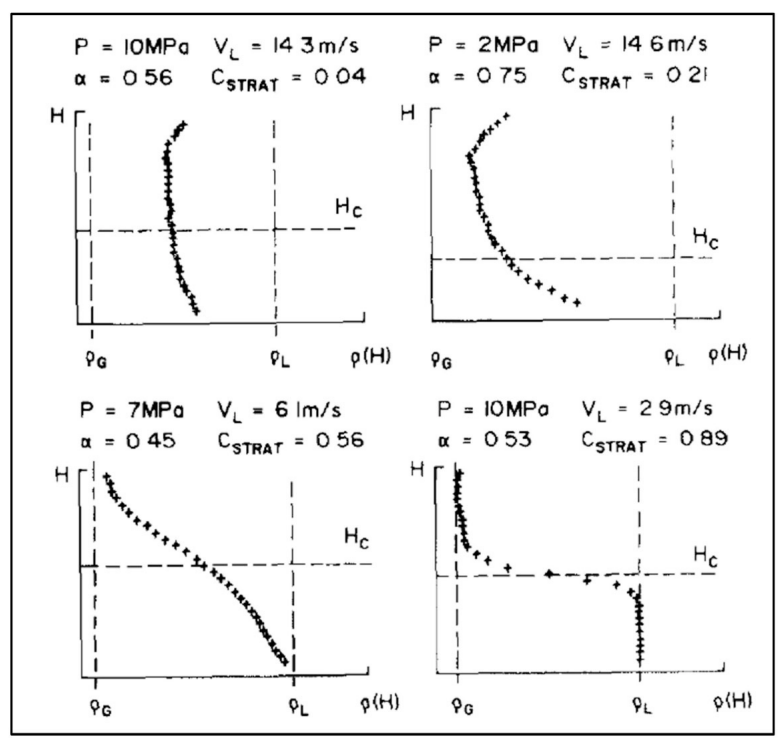

Fig. 8. Examples of Density Profile Measurements Performed in the SUPER MOBY DICK Horizontal Test Section: Upper Left is a Dispersed Bubbly Flow, Upper Right is an Annular Flow, Bottom Left is a Bubbly Flow with a Trend to Accumulate Bubbles at the Top of the Pipe, and Bottom Right is a Clear Stratified Flow

$\rho^{-}$and $\rho^{+}$are respectively the averaged density below and above Hc calculated using measured density profiles. For a flat density profile, $\mathrm{C}_{\mathrm{STRAT}}$ will approach zero. For a pure stratified flow regime with a flat free surface it will be nearly equal to 1 ; as there is always some scatter in the data due to noise and inaccuracy of the apparatus, the values 1 and 0 were never reached exactly.

However, one can consider that for $\mathrm{C}_{\mathrm{STRAT}}>0.75$ the flow is stratified and for $\mathrm{C}_{\mathrm{STRAT}}<0.5$, the flow is clearly not stratified.

As shown by Bestion [1] the experimental stratification rate $\mathrm{C}_{\text {STRAT }}$ is well correlated with the dimensionless velocity ratio $\mathrm{V}^{*}$. The thresholds between stratified and non-stratified flows were obtained from these data and are given in Table 1. A second rate of stratification R2 may be defined based on stability of bubbly flow.

\section{Data from METERO test facility}

In 2006 the new experiment METERO, dedicated to the flow regime transitions in horizontal pipe, started to provide an exhaustive database of measurements and videos to characterize the opponent hydrodynamic mechanisms responsible for horizontal water-air flow stratification. This experiment was part of the French NEPTUNE project
Table 1. Thresholds for Transition from Non-stratified to Stratified Flow Based on SMD Data

\begin{tabular}{c|c|c}
\hline \multicolumn{3}{|c}{ Rate of stratification : $\mathrm{R}_{2}$} \\
\hline $\mathrm{V}^{*}<40$ & $\mathrm{R}_{2}=1$ & $\begin{array}{c}\text { Stratification is } \\
\text { possible }\end{array}$ \\
\hline $40<\mathrm{V}^{*}<90$ & $\mathrm{R}_{2}=\frac{1}{50^{3}}\left(\mathrm{~V}^{*}-90\right)^{2}\left(2 \mathrm{~V}^{*}-30\right)$ & Transition \\
\hline $90<\mathrm{V}^{*}$ & $\mathrm{R}_{2}=0$ & $\begin{array}{c}\text { Bubbly flow remains } \\
\text { stable Stratification } \\
\text { is not possible }\end{array}$ \\
\hline
\end{tabular}

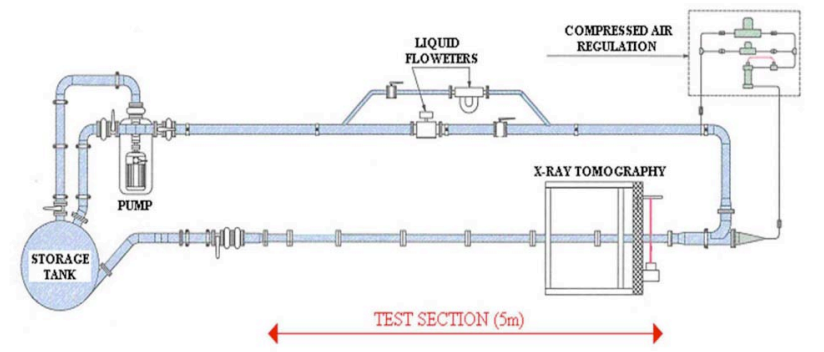

Fig. 9. METERO Test Facility.

(Guelfi \& al. [33], Bestion \& Guelfi [34]) for a multi-scale thermalhydraulic simulation platform, developed jointly by EDF and CEA associated with AREVA Nuclear Power (Areva NP) and the French Institute for Nuclear Safety (IRSN).

The METERO experiment (Marchand et al., [35], Bottin et al., [36], Bottin et al. [37]) test section (Figure 9) consists of a 5 meter long Plexiglas pipe, 0.1 meter in diameter, equipped with various measurement instruments, including hot film anemometry, optical probes and high speed video cameras.

In order to set the thresholds, a flow map is required. Thus, all the flow regimes that can exist in METERO were identified by visual observation and using high speed video. These flow regimes can be generated by varying the water superficial velocity from 0 to $5.3 \mathrm{~m} / \mathrm{s}$ and the gas superficial velocity from 0 to $0.125 \mathrm{~m} / \mathrm{s}$. Flow pattern maps have been plotted based on observations made using the high speed video camera. Then several CATHARE 2 computations were performed and the stratification index values $\mathrm{R} 2=0$ (bubbly flow remains stable), $\mathrm{R} 1=1$ (stratified flow is stable) corresponding to the CATHARE flow regime map have been plotted and compared to the METERO observations (Figure 10). The conclusion was first that the transition between stratified and intermittent regimes is rather well predicted $(\mathrm{R} 1=1)$. The second conclusion is that the transition between bubbly and intermittent regimes $(R 2=0)$ is not well captured by the model. Thus a new expression for the turbulent velocity scale was developed to try to improve this index. 


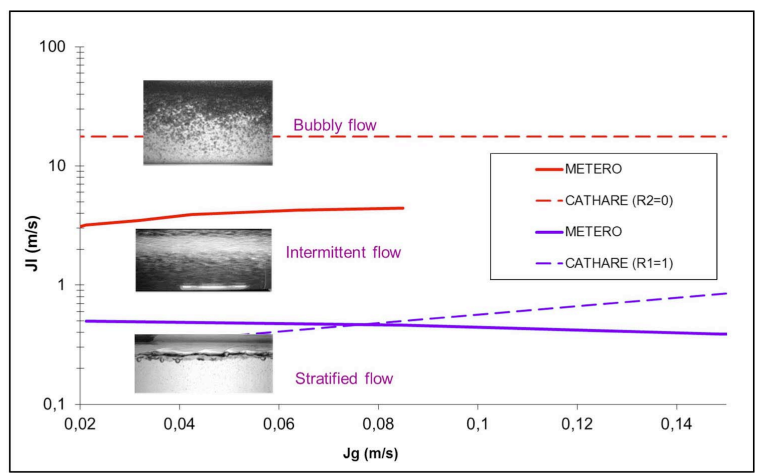

Fig. 10. METERO \& CATHARE Flow Maps

Table 2. Turbulent Kinetic Energy: Comparison of Experimental Data with Algebraic Model

\begin{tabular}{c|c|c|c|c}
\hline $\operatorname{Re}_{\mathrm{L}}$ & \multicolumn{4}{|c}{$4,2.10^{5}$} \\
\hline$\alpha$ & 0 & 0,007 & 0,02 & 0,06 \\
\hline Exp k & $1,1.10^{-1}$ & $1,2.10^{-1}$ & $1,3.10^{-1}$ & $1,4.10^{-1}$ \\
\hline Model k & \multicolumn{4}{|c}{$1,00.10^{-1}$} \\
\hline
\end{tabular}

The first step was to validate the algebraic model for the turbulent kinetic energy. Hot film anemometry was used to perform measurement of the axial and vertical components of the liquid velocity. From statistical treatment, the fluctuating/turbulent component velocity was extracted. And, with some assumptions, the turbulent kinetic energy was obtained. The measurements were performed along complete vertical profiles so the turbulent kinetic energy could be averaged spatially in order to be comparable to the model. These profiles were located at 5, 20 and 40 hydraulic diameters from the inlet. Only the last profile was used to validate the algebraic model of turbulence since it had been developed for established flows and assuming the flow being so at this place. Table 2 compares integrated averaged $\mathrm{k}$ data in single-phase and two-phase flows and the algebraic $\mathrm{k}$ model.

The algebraic model dedicated to single-phase flow gives quite an accurate value of the turbulent kinetic energy in single-phase flows and even in two-phase flow for the low void fraction cases that have been studied. The conclusion is that the algebraic model is capable of giving a correct order of magnitude for the turbulent kinetic energy.

Thresholds values were set as simply as possible and before any calculation, assuming that the flow was fully developed (bubbly or stratified) when the turbulent and bubble rising velocities are in a ratio of 2 (see Table 3 ).

The same CATHARE computations have been performed and the results are presented in the next figure.

The predicted first transition position (see the $\mathrm{R} 2=0$ line on Figure 11) is significantly improved. The model for R1 remains unchanged and so does the line $\mathrm{R} 1=1$. This result validates the approach.
Table 3. New Thresholds for the New Model

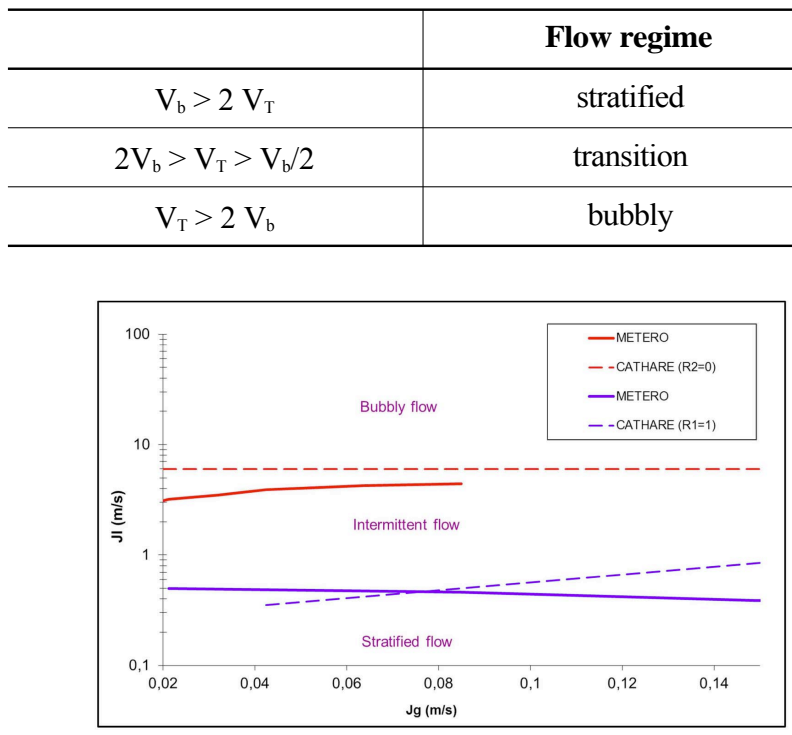

Fig. 11. METERO \& New CATHARE Flow Maps

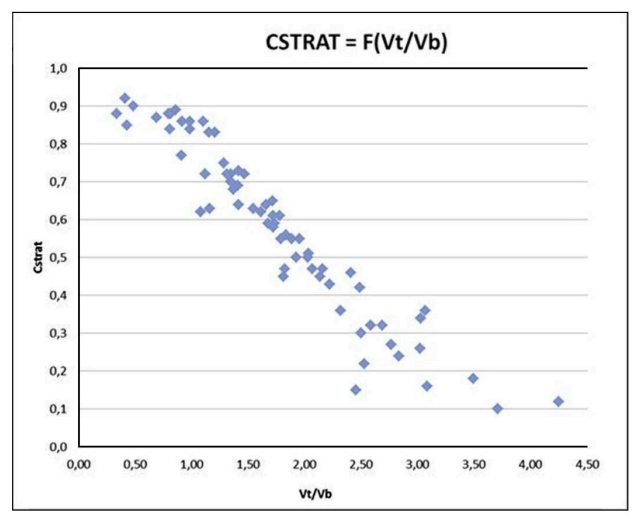

Fig. 12. $\mathrm{C}_{\text {Strat }}$ Versus the New Ratio Vt/Vb

One can plot the values of the new ratio as a function of $\mathrm{C}_{\text {STRAT }}$ obtained in SMD tests as in figure 12. One can observe that for $\mathrm{Vt} / \mathrm{Vb}>2$, the flow is clearly not stratified and that for $\mathrm{Vt} / \mathrm{Vb}<0.5$ the flow is clearly stratified. However one could extend the stratified domain up to $\mathrm{Vt} / \mathrm{Vb}<1$. Anyway this shows that the limit $\mathrm{Vt} / \mathrm{Vb}=2$ defining the limit of bubbly flow is in accordance with both METERO and SMD.

\section{PREDICTING FLOW REGIMES AND INTERFACIAL FRICTION}

\section{Flow regimes}

The prediction of the flow regime should be based on the local values of the principal variables. Based on these variables one can first look at the stability criteria for KelvinHemholtz instability and for the stability of bubbly flow to determine if a stable stratified flow is possible or if a 
stable bubbly flow is possible. Four cases are possible:

1. Stable stratified flow possible $\&$ stable bubbly flow impossible: in this case one can easily decide that the flow is stratified

2. Stable stratified flow impossible \& stable bubbly flow possible: in this case one can easily decide that the flow is bubbly

3. Stable stratified flow impossible \& stable bubbly flow impossible: in this case one can easily decide that the flow is intermittent (slug or plug flow)

4. Stable stratified flow possible \& stable bubbly flow possible: in this case one cannot easily decide what the flow regime is

This last case corresponds to flow conditions with rather high liquid velocity and rather low velocity difference between phases; this may occur in bubbly, slug and stratified flows. One should know the history of the flow to determine which regime is possible and this is not possible with only the six local variables of the two-fluid model. More advanced models may provide useful additional information. If a transport equation for the interfacial area was added it could clearly allow distinguishing stratified flow from bubbly flow. A multi-field model with two gas fields (continuous gas and dispersed bubbles) could also easily distinguish stratified flow from bubbly flow.

In the absence of knowledge of the history, the CATHARE 6-equation model preferred to select bubbly flow when both bubbly and stratified flow could be stable. A final rate of stratification $\mathrm{R}$ is defined which is the product of R1 (see section 6) and R2 (see section 5). This allows stratification only when stratified flow is stable and bubbly flow unstable. In PWR horizontal legs high liquid velocities are mainly encountered when pumps are still on. Therefore the flow at the inlet of the cold leg cannot be stratified due to strong perturbations in the pump. At the exit of the upper plenum there may be either a bubbly flow or a stratified flow with bubbles below the free surface depending on the position of a possible swell level in the upper plenum. In the latter case the CATHARE code may be partly wrong by predicting pure bubbly flow. However, this situation does not happen very often and no big discrepancy in Integral Effect Test prediction was observed which could be attributed to this weakness of the flow regime identification.

How to model flow regime transitions

Due to the geometry of the PWR circulating loops, flow regime transitions may occur in horizontal pipes, HL, IL,
CL since all horizontal pipes follow vertical upward or vertical downward flow. The prediction of the dynamics of the transition and of the length of pipe necessary to change the flow regime may be important when there is a break or a junction (e.g. pressurizer surge line) where phenomena will depend on the local structure of the flow. The transition from bubbly to stratified flow was observed in METERO and was found rather progressive with accumulation of bubbles in the upper part of the pipe, then with coalescence up to formation of a continuous gas layer. The transition from stratified to slug by Kelvin Helmholtz instability may be more violent. The instability is prepared by some wave formation at the free surface which increases in amplitude and there may be a very fast increase of the wave up to a liquid bridging. In principle a 1D 2-fluid model is able to predict the wave instability of shallow water waves. However this very fast transient is not supposed to be modelled by a $1 \mathrm{D}$ model, which uses combined space and time averaging. Therefore there must be a way to filter the process. This is the reason why a transition zone in the domain $0.25<\mathrm{Fr}<1$ was defined in the CATHARE code where some waves can be unstable. In this zone the interfacial friction coefficient is a weighted average of the low stratified flow coefficient and the high slug flow coefficient. The progressive increase of the interfacial friction will smooth the violence of the transition. This is qualitatively consistent with a time averaged physical model but there is no real quantitative control of the transition time period.

For the other transition from bubbly to stratified flow, a transition domain for the ratio $\mathrm{Vt} / \mathrm{Vb}$ was also defined in the CATHARE code where the interfacial friction changes progressively from a high value to a low value. Here again the predicted qualitative behaviour is consistent with the physics but there is no quantitative control of the transition time period and space extension.

\section{Interfacial friction}

In horizontal bubbly flow in large diameter pipes, there is a low axial pressure gradient and consequently the mean velocity of the bubbles and of the liquid are very close. It is not difficult to predict the right slip between phases which is nearly zero. A simple extrapolation of the interfacial friction from vertical bubbly flow would be convenient. For the same reasons extrapolating the interfacial friction from vertical slug flow would be convenient in horizontal flow.

For stratified flow the interfacial friction is much lower and there can be large velocity differences between

Table 4. How to Determine the Horizontal Flow Regime

\begin{tabular}{c|c|c}
\hline Stable stratified flow possible? & Stable bubbly flow possible? & Flow regime \\
\hline possible & impossible & Stratified flow \\
\hline impossible & impossible & Intermittent (slug, plug) \\
\hline impossible & possible & Bubbly flow \\
\hline possible & possible & Bubbly or stratified depending on history \\
\hline
\end{tabular}


phases. The interfacial friction coefficient depends a lot on the presence of waves. The TRACE code [5] adopted only a friction coefficient for stratified smooth regime. The RELAP-5 code also uses an interfacial friction coefficient for stratified flow which is similar to the wall friction (Blasius model). Several academic works may be found in the literature which correlated the interfacial friction based on experiments including wavy stratified flow. The friction coefficient depends on some gas Reynolds number or on a Froude number to take into account the roughness due to waves. However there may be a difficulty since the roughness due to waves may depend on the axial location along the pipe since waves are created and increase progressively in amplitude as shown in Figure 13.

The average liquid level $\mathrm{H}$ may be constant or quasiconstant while the amplitude of waves increases. This should produce an increasing interfacial friction coefficient but there is nothing in the principal variables of the twofluid model which can tell what the local amplitude of waves is. The time averaged values of the void fraction and of the phase velocities are constant. Therefore it is impossible to predict the axial increase of the interfacial friction when the wave amplitude increases. One can only adopt a homogeneous average friction coefficient which takes into account the presence of waves. However, for the same conditions, the average wave amplitude is smaller for a short pipe than for a longer pipe. It was considered that the models of the literature were not well adapted to the reactor HL, IL and CL since they were obtained for established flow conditions in very long pipes. Therefore the interfacial friction coefficient of the CATHARE code was derived from experiments which represented an intermediate leg [1] and a hot leg [8, 9]. This was supposed to be the best compromise.

One must notice that only the roughness of the waves which are not predicted by the code has to be taken into account in the averaged friction coefficient. As shown above only "shallow water waves" may be predicted by the two-fluid model due to the $p_{i} \partial \alpha / \partial z$ term which gives to the two-fluid equations the capabilities of the SaintVenant equations. Such waves have a wave length longer than twice the water depth. Shorter wavelength waves may be created at the free surface of the stratified flow which cannot be predicted by the two-fluid model -they are "deep water waves" - even if the time averaging was made on a very short time interval. At least all these deep

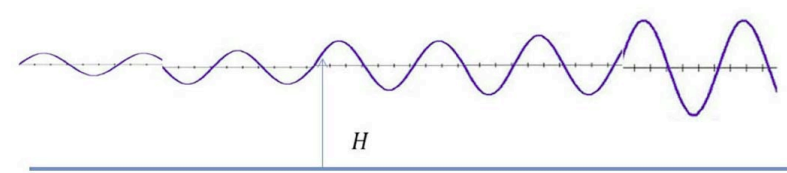

Fig. 13.Typical Formation of a Wavy Stratified Flow water waves should be taken into account by interfacial friction coefficients.

\section{PERSPECTIVE FOR FURTHER IMPROVEMENTS OF THE MODELLING}

\subsection{Dynamic Modelling of Interfacial Area and Turbulence}

The improvement of the flow regime transition and a better characterisation of the vertical void repartition may be obtained by a better modelling of both turbulent velocity scale and an average bubble diameter. In section 6 they are calculated using algebraic models which need some assumptions about flow establishment. Having a dynamic prediction of the turbulent scale and bubble size may improve the accuracy of the prediction of the transition from bubbly to stratified flow. Also, when both a stable stratified flow and a stable bubbly flow are possible when considering $\mathrm{Jv}, \mathrm{Jl}$ and $\alpha$ it is impossible to decide what the flow regime is without knowing the flow history, and the knowledge of the interfacial area may bring the missing information.

An algebraic turbulent model may give the asymptotical value reached by turbulent kinetic energy established flow. However, the evolution of two-phase flow structures (bubbles/pockets...) may induce modifications of the turbulence level and of bubble size and only transport equations would be able to capture these phenomena. For these reasons, transport equations for IA and turbulence were developed at CEA for the CATHARE 3 system code. They were first used in vertical bubbly flow [38], and they are used here for horizontal flow.

\section{Turbulence transport equation}

A one-dimensional two equations model has been developed for the turbulent kinetic energy (TKE) $k$ and its dissipation rate $\varepsilon$. The model is written:

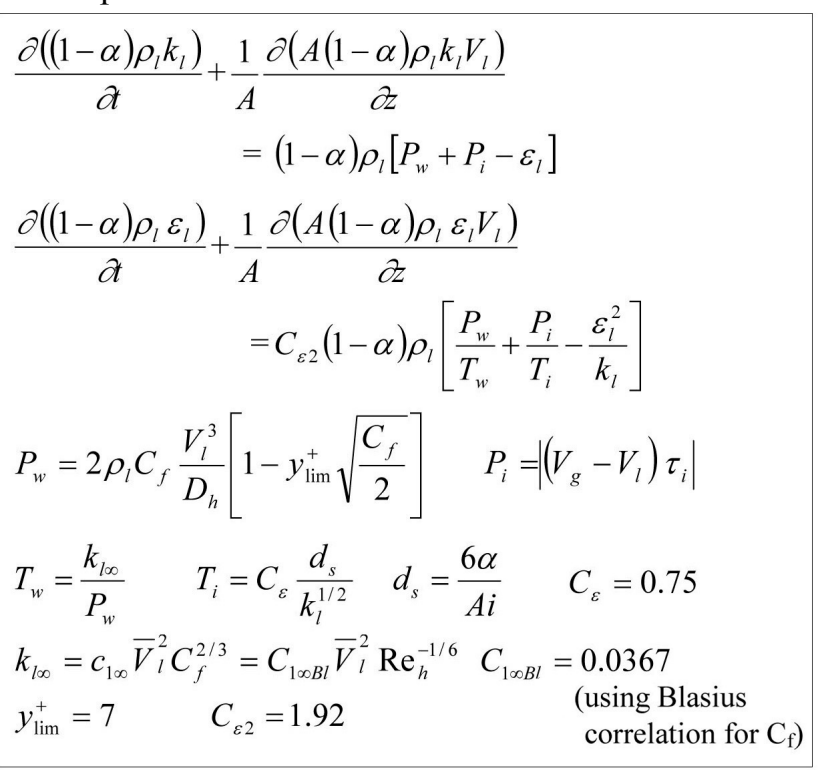


This model has been developed first using a porous medium approach in single-phase flow (Chandesris et al. [31], [39]). The modeling effort focused on the production terms in both equations. In the classical $(k, \varepsilon)$ turbulence model, the production is proportional to the mean velocity gradient and to the Reynolds stress. In a porous 1D approach, these quantities are unknown, thus the production term needs to be modeled. The single-phase turbulent production term $P_{k}$ was modeled as proportional to the power of the wall friction force (using a friction coefficient model) minus the viscous dissipation very close to the wall $\left(y_{+} \leq 7\right)$. The production of dissipation was modeled as proportional to the production term $P_{k}$ and divided by a time scale $T_{k}$. This time scale depends on the TKE at equilibrium $K_{k \infty}$ which is also used as the previously mentioned algebraic model. When the temporal and spatial derivatives tend to zero, the model gives this asymptotical equilibrium value, where both transport and algebraic model are in agreement. In single-phase flow, this model has been validated successfully against various experimental data and DNS results (see [31] and [39]). Then, this approach was extended to two-phase flow with modeling of the twophase production term $P_{k i}$ (Serre et al. [38]): it can be estimated using the power of the interfacial friction force (slip velocity multiplied by the interfacial friction force). It was validated successfully in vertical bubbly to churn flows using the DEDALE experiment data (Grossetete, [40]).

In horizontal bubbly flow, METERO data are used to validate the model. In such horizontal flow, the bubbles have nearly the same mean velocity as the liquid. Thus, the turbulence induced by the bubbles' motion comes more from their random fluctuations than from a mean slip in the liquid. Therefore the previous modeling of two-phase production has to be revisited. It was realized as simply as possible by correlating it with the liquid velocity (to the power of 3 for dimensional reasons) and the void fraction (to the power of $\mathrm{n}$ to be obtained empirically): $P_{k i a} \propto \alpha^{n} V_{l}^{3}$. Experimental fitting gave this final expression for the production in horizontal bubbly flows:

$$
P_{i}=0.13 \alpha^{1 / 5} V_{l}^{3}
$$

Using this expression, the final TKE is captured in bubbly flow as illustrated in Figure 14. The intermediate state of TKE $(x=2000 \mathrm{~mm})$ is overestimated for the following reason: the mean production term is the single-phase one which is calculated using the friction coefficient for established flows while friction is not established at this section of METERO, since the boundary layers are developing from the inlet to the last measurement point at $4000 \mathrm{~mm}$. Nevertheless, this turbulent model is capable of giving a good estimation of turbulent kinetic energy even in bubbly flows. More validation would be appreciable especially for intermittent flows and stratified ones.

One may notice that the liquid turbulence plays a dominant role in the direct contact condensation at ECCS injections. It was found that all sources of turbulence,

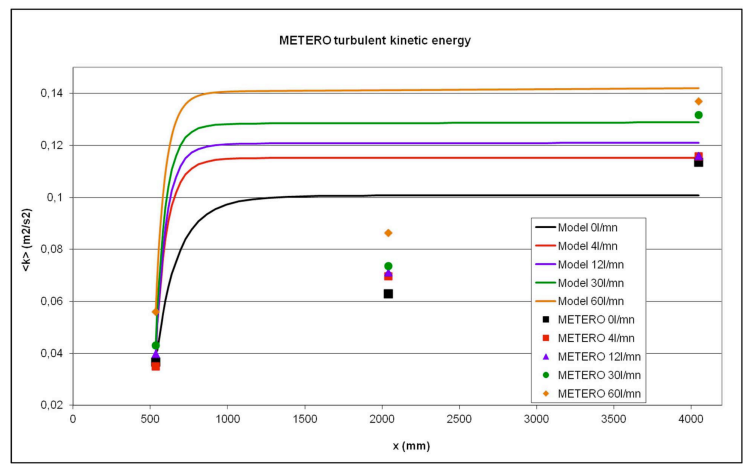

Fig. 14. Spatial Evolution of TKE. Liquid Flow Rate $125 \mathrm{~m}^{3} / \mathrm{h}$, Air Flow Rates Indicated in the Legend.

including the turbulence due to safety injection jets, had to be taken into account in the condensation modeling (Janicot \& Bestion [12]). It could be more convenient to use a dynamic modeling of turbulence to better model these effects in all situations.

\section{Interfacial area transport equation}

Developing a transport of interfacial area (TIA) equation in a system code is quite different from developing such a model in a CMFD code. A lot of work was devoted to the modelling of TIA in CMFD codes for dispersed flows, but the other flow regimes that system codes must also calculate remain terra incognita. The use of several equations to model polydispersion is not reasonable in a system code which must model all the existing flow regimes in many geometrical configurations. The required modelling work of TIA for all the flow regimes that can occur in reactor, and our ignorance of many of the physical phenomena that govern the evolution of IA, lead to the adoption of a different strategy for TIA modelling in system codes than in CMFD codes (Bestion, [41]). Some of the conclusions drawn by Bestion for the TIA modelling strategy for system codes were the following:

- To accept some empiricism in TIA equation closure laws.

- Identify some flow situations in reactor TH where TIA may improve system code predictions and focus R\&D on these reactor issues.

- Define a step by step strategy for TIA implementation in system codes.

- Try to simplify closure laws so that existing SETs without IA measurement may also be used for TIA validation.

In this context, METERO gives useful data for TIA modelling in horizontal flow from bubbly to stratified flow. Thanks to two sensor and four sensor optical probes, the void fraction and IA is measured and then averaged spatially for the 1D modelling in system codes. A simple relaxation model has been developed for bubbly flows [36]:

$$
\frac{d A i}{d t}=\frac{A i-A i_{\infty}}{\tau}
$$


where $A i_{\infty}$ is the asymptotic value and $\tau$ the relaxation time scale to reach this asymptotic value.

These two quantities contain the physics of the flow and must be modelled using experimental data.

The asymptotic value model was written after noticing that the (averaged) IA depends linearly on the superficial gas velocity (for a given superficial liquid velocity) ([36]):

$$
A i_{\infty}=\left(10630-11223 \frac{J_{L}}{J_{L}+0.59}\right) . J_{G}+2.59 J_{L}-10.68
$$

This behaviour was noticed by J.M. Delhaye for vertical flows ([42]), which is encouraging for the generalisation of this kind of modelling:

$$
A i_{\infty}=\left(10710-10100 \frac{J_{L}}{J_{L}+0.081}\right) . J_{G}
$$

The time scale is modelled as the vertical travelling time for bubbles to reach the top of the pipe where they can coalesce, decreasing IA [36]:

$$
\tau=\frac{D_{H}}{V_{b}}
$$

Using this model, some comparisons with the METERO results have been performed for one liquid flow rate and four gas flow rates. Except for one point, the agreement between the model and data is very good as illustrated on Figure 15.

This model could be generalised, adding other phenomena:

$$
\frac{d A i}{d t}=\frac{A i-A i_{\infty}}{\tau}+S_{\text {boiling }}+S_{\text {flashing }}-S_{\text {collapse }} \cdots
$$

Many others experiments are necessary to develop a more general model for TIA, valid for all the flow regimes of interest in a reactor. However, in HL, IL and CL boiling, flashing and collapse do not play an important role.

Status of the dynamic IA and turbulence modelling

The attempts to develop a dynamic model of both turbulence and interfacial area in vertical bubbly flow [38] and here in horizontal bubbly flow have proven the interest of the approach but much more development and validation work are necessary before their implementation in an industrial version of a system code.

It is shown that both the bubble size and the turbulence can significantly change from the entrance of a horizontal pipe to the exit, and the transition time from bubbly to stratified flow may be affected by these changes.

However, the knowledge of IA may not be sufficient to transport the information required to decide what flow regime takes place when both a stable stratified flow and a stable bubbly flow are possible when considering $\mathrm{Jv}, \mathrm{Jl}$ and $\alpha$. If the transition from bubbly to stratified flow is in progress, there may be a continuous gas layer at the top and dispersed bubbles below the free surface. The interfacial area remains high as long as there are bubbles even if most of the gas flowrate is in the continuous gas layer. Then TIA

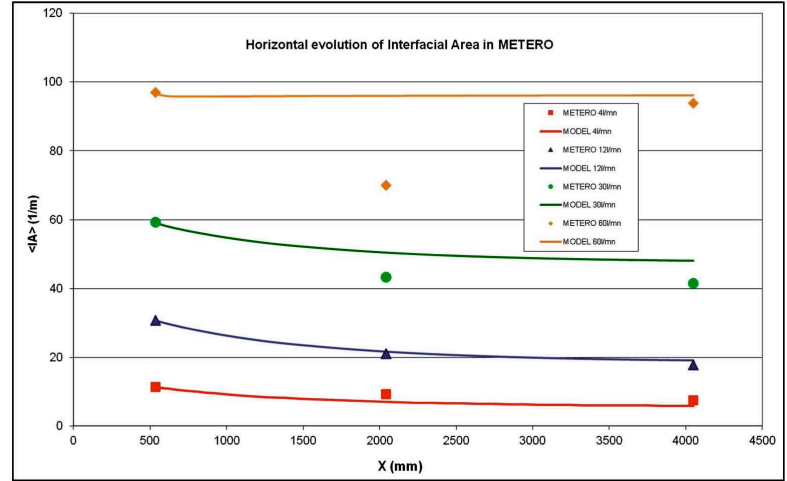

Fig. 15. Spatial Evolution of IA. Liquid Flow Rate $125 \mathrm{~m}^{3} / \mathrm{h}$, Air Flow Rates Indicated in the Legend.

is not sufficient to see that stratification is in progress and /or nearly achieved. In such a condition multi-field modeling may bring the required information.

\subsection{Multi-field Modelling}

The separation of the gas phase into two fields, one for the continuous gas in the case of stratified or annular flow and one for the dispersed bubbles or slugs, may be of great interest for the following reasons:

1. The knowledge of the two gas field volume fractions keeps the history of the flow regime since stratified flow exists only when the continuous gas fraction is not zero

2. The knowledge of the two gas field volume fractions characterizes more precisely the vertical void repartition in bubbly flow, in stratified flow, and during the transition from bubbly to stratified flow when both gas fields are present. This vertical void repartition influences the behaviour at a break or at a junction (e. g. pressurizer surge line).

3 . The prediction of the progressive transition from bubbly to stratified flow may be treated mechanistically by modelling the transfer between fields and the time period and space extension of the transition may be physically controlled. The METERO tests $[35,36,37]$ have sufficient information to derive models for this transfer.

4. Complex situations with totally different velocities of bubbles and continuous gas may be better modelled with two separate velocities.

The separation of the liquid phase into two fields, one for the continuous liquid in the case of stratified or annular flow and the other for dispersed droplets may be of great interest for the following reasons:

1. The knowledge of the two liquid field volume fractions keeps the history of the flow regime since droplets may come from another reactor component (e.g; the pressure vessel) whereas the continuous liquid field may result from a long period of droplet deposition (this may occur during the reflooding 
phase of a LBLOCA) or it may come from downstream of the horizontal pipe in reflux condensation.

2. The knowledge of the two liquid field volume fractions allows more precise characterization of the vertical void repartition in stratified flow and stratified-mist flow. This vertical void repartition influences the behaviour at a break or at a junction (e. g. pressurizer surge line).

3. The prediction of the progressive transition from stratified-mist flow to pure stratified flow by droplet deposition or from pure stratified flow to stratifiedmist flow may be treated mechanistically by modelling the transfer between fields and the time period and space extension of the transition may be physically controlled.

4. Complex situations with totally different droplet and continuous liquid velocities may be better modelled with two separate velocities. In particular it was found that the transit time of droplets between the core and the steam generators during the Reflooding of a LBLOCA could play an important role in the steam binding process for the damping or not of gravity oscillations between core and donwncomer (Valette et al. [10]). This transit time is not respected with the two-fluid model since the unique liquid velocity is an average between the droplet velocity and the continuous liquid velocity.

5. The prediction of the two liquid field temperatures may predict direct contact condensation at ECCS injection in case of stratified-mist flow more precisely. Even if the heat transfer coefficients at the free surface and for the droplets were perfectly modelled, the two-fluid model with only one average liquid temperature may not correctly predict the respective heat transfers since the two temperatures may become very different: the droplet temperature reaches saturation very soon whereas a continuous liquid may keep a high subcooling a long time. However such situations, with entrainment of droplets at ECCS injection, are not encountered very often and have only been observed during very short periods in LBLOCA simulations at the beginning of accumulator injection.

\section{CONCLUSION}

The classical two-fluid six-equation one-pressure model as it is implemented in the CATHARE code was presented and it was shown that the axial effects of gravity forces may be correctly taken into account even in case of change of the cross section area or of the pipe orientation. It was also shown that it can predict both fluvial and torrential flow with a possible hydraulic jump. The shallow water waves may be predicted but the presence of small wavelength "deep water waves" should be treated as a free surface roughness in the interfacial friction coefficient for a stratified flow regime.
Since phase stratification plays a dominant role, the Kelvin-Helmholtz instability and the stability of bubbly flow regime were considered. A transition criterion based on a stability analysis of shallow water waves may be used to predict the Kelvin-Helmholtz instability. Recent experimental data obtained in the METERO test facility were analysed to model the transition from bubbly to stratified flow regime. The prediction of the actual flow regime may be obtained from these two criteria except when both stratified flow and bubbly may be found stable. For this situation the two-fluid model cannot decide what the flow regime is whereas a multi-field could easily determine the flow regime.

The use of a transition domain between bubbly and stratified flow or between stratified and slug flows provides a progressive change of flow regime. Then the predicted qualitative behaviour is consistent with the physics but there is no quantitative control of the transition time period. Here again a multi-field model could model the transition and the time period and space extension of the transition may be physically controlled. A dynamic modelling of liquid turbulence and bubble interfacial area could be used to model precisely the time scales associated with the transition from bubbly to stratified flow. It could be more convenient to use a dynamic modeling of turbulence to better model interfacial heat transfers in all situations, particularly for direct contact condensation.

A 4-field model with a continuous liquid field, a continuous gas field, a dispersed bubble field, and a dispersed droplet field, has many capabilities to improve the prediction of flow regimes, the dynamics of flow regime transitions, the mechanical behaviour and the interfacial transfers in complex situations when a phase is split into two fields. In addition it could provide a better characterisation of the flow with a more precise vertical void repartition. Threefield models with two liquid fields are already being implemented in CATHARE-3 and SPACE codes. Four- models already exist in the petroleum industry for liquid-gas flows in pipelines and could probably be implemented in system codes in the medium term future.

\section{ACKNOWLEDGEMENTS}

The authors are grateful to CEA, AREVA, EDF and IRSN who financed the NEPTUNE project for a multiscale thermalhydraulic platform, to the members of the NEPTUNE project team, and particularly to M. Marchand, M. Bottin and J. P. Berlandis who performed and analyzed the METERO tests which are presented in this paper.

\section{NOMENCLATURE}

\section{Latin letters}
A Cross section area
$\mathrm{Ai}$ Interfacial Area
$\mathrm{C}_{\mathrm{d}}$ drag coefficient
$\mathrm{C}_{\mathrm{f}}$ friction coefficient 
$\mathrm{D}_{\mathrm{h}}$ hydraulic diameter

$\mathrm{d}_{\mathrm{s}} \quad$ Sauter diameter

g gravity

J superficial velocity

$\mathrm{k}$ turbulent kinetic energy

$£ \quad$ Laplace scale

$\mathrm{P}$ Pressure or production

Re Reynolds number

$t$ time

$\mathrm{S}$ surface

$\mathrm{T}$ time scale

$\mathrm{V}$ mean velocity

Wi interfacial velocity

$\mathrm{y}^{+}$wall non dimensional coordinate

$\mathrm{z}$ curvilinear abscissa along tube axis

\section{Greek letters}

$\alpha$ void fraction

$\delta$ bubble diameter

$\varepsilon \quad+/-1$ or turbulence dissipation

$\rho$ density

$\sigma$ surface tension

$\tau$ friction

$\theta$ pipe inclination angle

$\begin{array}{ll}\text { Subscript } \\ \text { b } & \text { bubble } \\ \text { f } & \text { friction } \\ \text { g } & \text { gas } \\ \text { V } & \text { vapor } \\ \text { i } & \text { interface } \\ \text { k } & \text { phase } \mathrm{k} \\ \text { l } & \text { liquid } \\ \mathrm{m} & \text { mixture } \\ \mathrm{T} & \text { turbulent } \\ \mathrm{W} & \text { wall } \\ \infty & \text { asymptotic value }\end{array}$

\section{REFERENCES}

[1] D. Bestion, The physical closure laws in the Cathare code, Nuclear Engineering \& Design, 124 (1990) 229-245

[2] O. Antoni, M. Farvacque, G. Geffraye (1), D. Kadri, G. Lavialle, B. Rameau, A. Ruby, CATHARE 2 V2.5_2 : a Single Version for Various Applications, 13th International Topical Meeting on Nuclear Reactor Thermal Hydraulics (NURETH-13), Kanazawa City, Ishikawa Prefecture, Japan, September 27-October 2, 2009

[ 3 ] P. Emonot, A. Souyri, J.L. Gandrille, F. Barré, CATHARE 3: A new system code for thermal-hydraulic in the context of the NEPTUNE project, 13th International Topical Meeting on Nuclear Reactor Thermal Hydraulics (NURETH-13), Kanazawa City, Ishikawa Prefecture, Japan, September 27October 2, 2009

[4] RELAP5/MOD3 Code Manual, Volume I : Code Structure, System Models and Solution Methods, NUREG/CR-5535, 1995

[ 5] TRACE-V5.0, Theory Manual, 2007, TRACE V5.0 User
Manual, 2007, TRACE V5.0 Assessment Manual, 2007

[6] G. Geffraye, P. Bazin, P. Pichon, and A. Bengaouer, CCFL in Hot Legs and Steam Generators and Its Prediction with the CATHARE code, Proceedings of the 7th International Topical Meeting on Nuclear Reactor Thermal Hydraulics, September10-15, 1995, pp.815-826

[ 7 ] G. Geffraye, S. Laroche, Analysis of the MHYRESA HotLeg entrainment tests, Ninth International Topical Meeting on Nuclear Reactor Thermalhydraulics (NURETH 9), San Francisco, California, October 3-8, 1999

[ 8 ] G. Ratel, G. Geffraye, Analysis with CATHARE code of the stratified flow regime in the MHYRESA Hot Leg entrainment tests, 38th European two-phase Flow Group meeting, Karlsruhe, Germany, 29-31 May, 2000

[9] G. Geffraye, B. Faydide, S. Laroche, G. Ratel, Analysis of the MHYRESA Hot-Leg entrainment tests, 8th International Conference on Nuclear Engineering, Baltimore, MD, USA, April 2-6, 2000

[10] M. Valette , J. Pouvreau, D. Bestion, P. Emonot, Revisiting Large Break LOCA with the CATHARE 3 Three Field Model, Nuclear Engineering and Design, Volume 241, Issue 11, November 2011, Pages 4487-4496

[11] D. Bestion, Fluid Dynamic effects in the fuel element top nozzle area and two-phase flow in piping junctions, Proceedings of the Seminar on the Commission Contribution to Reactor Safety research, edited by W. Krishner, Elsevier Applied Science, Varese Italy, 20-24 november 1989

[12] A. Janicot, D. Bestion, Condensation modelling for ECC injection, Nuclear Engineering \& Design, 145 (1993) 37-45

[13] M. Murase, A. Tomiyama, D. Lucas, I. Kinoshita, Y. Utanoharaa and C. Yanagia, Correlation for countercurrent flow limitation in a PWR hot leg, Journal of Nuclear Science and Technology, Volume 49, Issue 4, 2012

[14] D. Bestion, Status and perspective for a multiscale approach to Light Water reactor thermalhydraulic simulation, NURETH 14 , to be published in topical Issue of Nuclear Engineering and Design, 2012

[15] D. Bestion, Applicability of two-phase CFD to nuclear reactor thermalhydraulics and elaboration of Best Practice Guidelines, presented at CFD4NRS-3, Washington, september 2010, in press to be published in a Special Issue of Nuclear Engineering and Design, 2012

[16] D. Bestion, P. Coste, B. Niceno, S. Mimouni, Two-phase CFD: the various approaches and their applicability to each flow regime, Multiphase Science and Technology, Volume 23, Issue 2-4, 2011

[17] D. Bestion, The difficult challenge of a two-phase CFD model for all flow regimes, CFD4NRS-4, Dae Jeon, Korea, September 10-12, 2012

[18] P. Vernier \& J.M. Delhaye, General Two-Phase Flow equations Applied to the thermohydrodynamics of Boiling Water reactors, Energie Primaire, Vol. 4, no. 1-2

[19] J.M. Delhaye, M. Giot, M.L. Riethmuller, Thermohydraulics of Two-Phase systemsfor Industrial design and Nuclear engineering, Hemisphere Publishing Corporation, McGraw hill Book Company, 1981

[20] M. Ishii, Thermo-Fluid Dynamic theory of Two-Phase Flow, Eyrolles, Paris, 1975

[21] I. Tiselj and C. Samuel Martin, Two-fluid model for 1D simulations of water hammer induced by condensation of hot vapor on the horizontally stratified flow, 7th International 
Conference on Multiphase Flow, ICMF 2010, Tampa, FL USA, May 30-June 4, 2010

[22] I. Tiselj, Slug modelling with 1D two-fluid model, 14th International Topical Meeting on Nuclear Reactor Thermalhydraulics, NURETH-14 Toronto, Ontario, Canada, September 25-30, 2011

[23] A. E. Dukler, Y. Taitel, Gas-Liquid Flow Pattern, Multiphase Science and Technology, Ed. J. F. Hewitt, J. M. Delhaye, N. Zuber, Vol. 2, pp. 1-94, Hemisphere, Washington, 1986

[24] G. B. Wallis, J. E. Dobson, The onset of slugging in Horizontal Stratified Air-Water Flow, International journal of Multiphase Flow, Vol. 1, pp. 173-195, 1973

[25] J. M. Manhane, G. A. Gregory, K. Aziz, Intern. Journal of Multiphase Flow, vol. 1, pp. 537-553, 1974

[26] Y. Taitel, A. E. Dukler, A Model for predicting Flow Regime Transitions in Horizontal and Near-horizontal GasLiquid Flow, AICH Journal 22, (1), pp. 47-66

[27] D. Barnea, O. Shoham, Y. Taitel, A.E. Dukler, Flow Pattern Transitions for Downward Inclined two-phase flow; Horizontal to vertical, Chemical Engineering Sc. Vol. 37, ํ5, pp. 735-740

[28] D. Barnea, O. Shoham, Y. Taitel, Flow Pattern Transitions for Gas-Liquid Flow in Horizontal and inclined pipes, Intern. Journal of Multiphase Flow, vol. 6, pp. 217-225, 1980

[29] D. Bestion, J.C. Micaelli, A two-fluid stratified model suitable for Pressurized Water Reactor safety code, 4th Miami International Symposium on multiphase transport and particulate phenomena, 15-17 December 1986, Miami Beach, Florida, USA

[30] F. de Crecy, Modeling of Stratified two-phase flow in pipes, pumps and other devices, Int. J. Multiphase Flow, Vol. 12, pp 307-323, 1986

[31] M. Chandesris, G. Serre, P. Sagaut, 2006, A macroscopic turbulence model for flow in porous media suited for channel, pipe and rod bundle flows, Int. J. Heat Mass Transfer, vol. 49, pp 2739-2750.

[32] A Memponteil, "Destratification in steam water flow", European Two Phase Flow Group Meeting, Brussels, 1988.
[33] A. Guelfi, D. Bestion, M. Boucker, P. Boudier, P. Fillion, M. Grandotto, J.M. Herrard, E. Hervieu, P. Peturaud, NEPTUNE A new Software Platform for advanced Reactor Thermalhydraulics, Nuclear Science and Engineering, 156, 282-324, 2007

[34] D. Bestion, A. Guelfi, "Status and perspective of twophase flow modelling in the NEPTUNE Multiscale thermalhydraulic platform for nuclear reactor simulation », Nuclear Engineering and Technology, 2005, Vol. 16, Nos 1-3, pp 1-5.

[35] M. Marchand, M. Bottin, J-P. Berlandis, G. Serre and E. Hervieu, "Experimental Investigation and Physical Modeling of a Two-Phase Bubbly Flow in Horizontal Pipe", ICMF 7, USA, June 2010.

[36] M. Bottin, M. Marchand, J.P. Berlandis, E. Hervieu, F. Gateau and G. Serre, "Experimental Investigation and Modelling of a Two-Phase Bubbly Flow in Horizontal Pipe", NUTHOS 8, China, 2010.

[37] M. Bottin, J. P. Berlandis, E. Hervieu, M. Lance, M. Marchand, O. C. Öztürk and G. Serre, "Experimental Investigation and Physical Modeling of a Two-Phase Bubbly Flow in Horizontal Pipe", to be submitted to Int. J. of Multiphase Flow.

[38] G. Serre, D. Bestion, "Progress in improving two-fluid model in system code using turbulence and interfacial area equations", NURETH 11, Avignon, France, 2005.

[39] M. Chandesris, G. Serre, "One dimensional averaged (Kepsilon) turbulence model applied to channel, pipe and rod bundle flows", NURETH 11, Avignon, France, 2005.

[40] C. Grossetete. « Caractérisation expérimentale et simulations de l'évolution d'un écoulement diphasique à bulles ascendant dans une conduite verticale », $\mathrm{PhD}$ thesis, Ecole Centrale de Paris. 05/19/1995

[41] D. Bestion, Perspective of transport of interfacial area in system codes, 2010 ANS Winter Meeting, November 7$11,2010 \cdot$ Las Vegas, NV

[42] J. M. Delhaye \& P. Bricard, Interfacial area in bubbly flow: experimental data and correlations, Nucl. Eng. \& Des. Vol. 151, 65-77 (1994). 